\title{
A mitochondrial genome sequence of a hominin fromSima de los Huesos
}

\author{
Matthias Meyer ${ }^{1}$, Qiaomei Fu ${ }^{1,2}$, Ayinuer Aximu-Petri ${ }^{1}$, Isabelle Glocke ${ }^{1}$, Birgit Nickel ${ }^{1}$, Juan-Luis Arsuaga ${ }^{3,4}$,

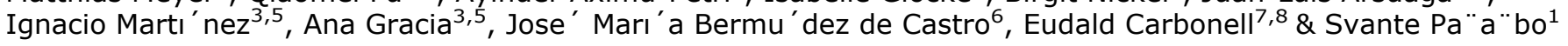

\begin{abstract}
Excavations of a complex of caves in the Sierra de Atapuerca in northern Spain have unearthed hominin fossils that range in age from the early Pleistocene to the Holocene'. One of these sites, the 'Sima de los Huesos' ('pit of bones'), has yielded the world's largest assemblage of Middle Pleistocene hominin fossils ${ }^{2,3}$, consisting of at least 28 individuals ${ }^{4}$ dated to over 300,000 years ago $^{5}$. The skel-etal remains share a number of morphological features with fossils classified asHomo heidelbergensis and also display distinct Neanderthal-derived traits $^{6-8}$. Here we determine an almost complete mitochon-drial genome sequence of a hominin from Sima de los Huesos and show that it is closely related to the lineage leading to mitochondrial genomes of Denisovans $^{9,10}$, an eastern Eurasian sister group to Neanderthals. Our results pave the way for DNA research on homi-nins from the Middle Pleistocene.
\end{abstract}

The Sima de los Huesos site (see Fig. 1 for a map) is located at the foot of a $13 \mathrm{~m}$ vertical shaft, about $30 \mathrm{~m}$ below the surface and $500 \mathrm{~m}$ from the closest current entrance to the karst system ${ }^{11}$. Humidity at the site is close to saturation, temperature in the cave is constant around 10.6 uC and the fossils have been protected from major disturbances since deposition ${ }^{12}$. The Sima de los Huesos is also noteworthy because it has provided unique evidence of long-term DNA survival. DNA preservation in the site was first proposed based on enzymatic amplification of a few short mitochondrial DNA (mtDNA) fragments from Middle Pleistocene cave bear remains ${ }^{13}$. Recently, improvements in

DNA extraction ${ }^{14}$ and library preparation ${ }^{10}$ techniques for highly

degraded ancient DNA have enabled the retrieval of a complete mitochondrial genome of a cave bear (Ursus deningeri) found with the hominin remains in the cave ${ }^{14}$. DNA preservation for hundreds of thousands of years has otherwise been documented only under permafrost conditions ${ }^{15,16}$

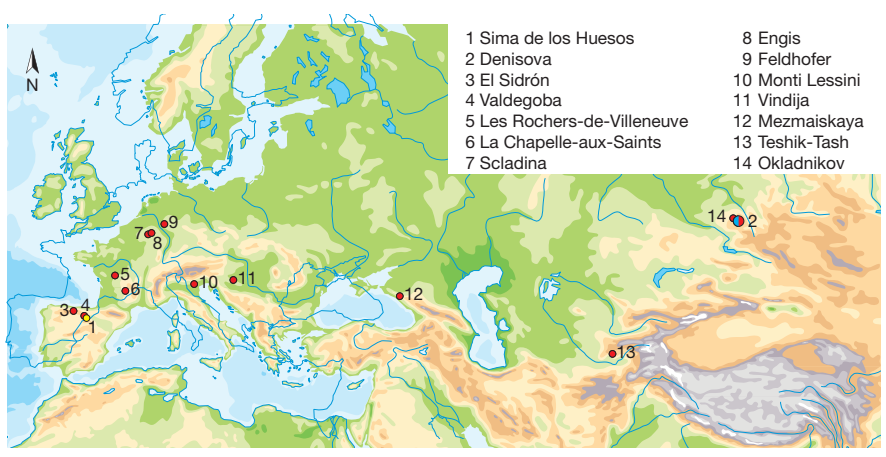

Figure 1 Location of the Middle Pleistocene site of Sima de los Huesos (yellow) as well as Late Pleistocene sites that have yielded Neanderthal DNA (red) and Denisovan DNA (blue).
To investigate whether DNA may also be preserved in the hominin remains, we obtained several samples of bone, totalling $1.95 \mathrm{~g}$, by drilling holes into the breaks of a femur (Femur XIII, ref. 17) excavated in three parts, one in 1994 and the other two in 1999 (Fig. 2). DNA was isolated using a recently published silica-based method ${ }^{14}$ and converted into 77 libraries for sequencing ${ }^{10,18}$ (Extended Data Table 1). Following library amplification, we first characterized a subset of the libraries by shallow shotgun sequencing on Illumina's MiSeq platform (Extended Data Fig. 1). Overlapping paired-end reads were merged to reconstruct full-length molecule sequences and mapped against the human gen-ome using BurrowsWheeler alignment (BWA) ${ }^{19}$. For most libraries, fewer than $0.1 \%$ of the sequences could be confidently aligned to the human genome (Extended Data Table 2), but 21 libraries yielded pro-portions of aligned sequences that were high enough (between $0.1 \%$ and $8.4 \%$ ) to investigate the frequencies of $\mathrm{C}$ to $\mathrm{T}$ substitutions at sequence ends, which are increased in authentic ancient DNA due to accelerated cytosine deamination in single-stranded overhangs ${ }^{20-22}$. However, in no case did $\mathrm{C}$ to $\mathrm{T}$ substitution frequencies exceed $3 \%$ at 59 ends and $6 \%$ at 39 ends (Extended Data Table 2), indicating that those libraries that are rich in human DNA are dominated by present-day human contamination.

We next enriched all libraries for mtDNA, using a probe set based on a present-day human sequence. An initial inspection of the isolated sequences revealed the closest similarities to the mtDNA of a Denisovan, an extinct archaic group related to Neanderthals ${ }^{9}$. Therefore, the libraries were additionally enriched with probes based on the Denisovan mtDNA $^{23}$. Sequencing was performed on Illumina's HiSeq 2500 platform from both ends, and overlapping reads were merged and aligned to the human reference mtDNA. Sequences with identical start and end coordinates, which often represent amplification products of the same starting molecules, were fused to create consensus sequences, and sequences shorter than 30 base pairs (bp) were discarded. The enriched libraries yielded a sufficient number of mitochondrial sequences to estimate the frequencies of $\mathrm{C}$ to $\mathrm{T}$ substitutions. These varied widely among the libraries, ranging from $1 \%$ to $45 \%$ at 59 ends, and from $2 \%$ to $47 \%$ at 39 ends (Extended Data Table 1). In agreement with the shotgun

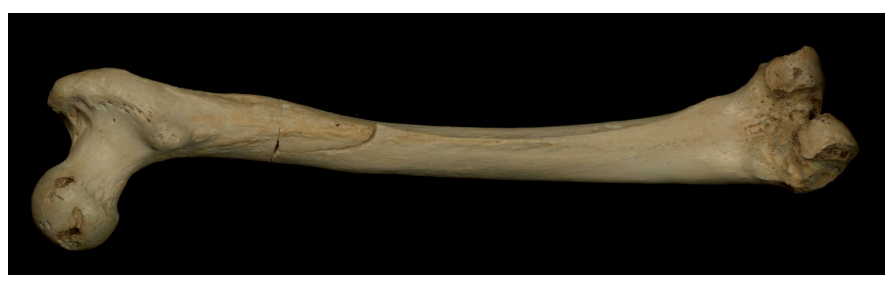

Figure 2 | Femur XIII reassembled from three parts after sampling. The natural fractures are visible in the proximal third of the femur.

Department of Evolutionary Genetics, Max Planck Institute for Evolutionary Anthropology, Deutscher Platz 6, 04103 Leipzig, Germany. ${ }^{2}$ Key Laboratory of Vertebrate Evolution and Human Origins of Chinese Academy of Sciences, Institute of Vertebrate Paleontology and Paleoanthropology, Chinese Academy of Sciences, Beijing 100044 , China. ${ }^{3}$ Centro de Investigacio'n Sobre la Evolucio'n y Comportamiento Humanos, Universidad Complutense de Madrid-Instituto de Salud Carlos III, 28029 Madrid, Spain. ${ }^{4}$ Departamento de Paleontologi'a, Facultad de Ciencias Geolo' gicas, Universidad Complutense de Madrid, 28040 Madrid, Spain. ${ }^{5} \mathrm{~A}^{\prime}$ rea de Paleontologi'a, Depto. de Geografı'a y Geologı' a, Universidad de Alcala', Alcala' de Henares, 28871 Madrid, Spain. ${ }^{6}$ Centro Nacional de

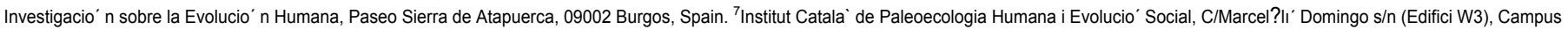
Sescelades, 43007 Tarragona, Spain. ${ }^{8}$ 'Area de Prehisto 'ria, Dept. d'Histo` ria i Histo` ria de l'Art, Univ. Rovira i Virgili, Fac. de Lletres, Av. Catalunya, 35,43002 Tarragona, Spain. 

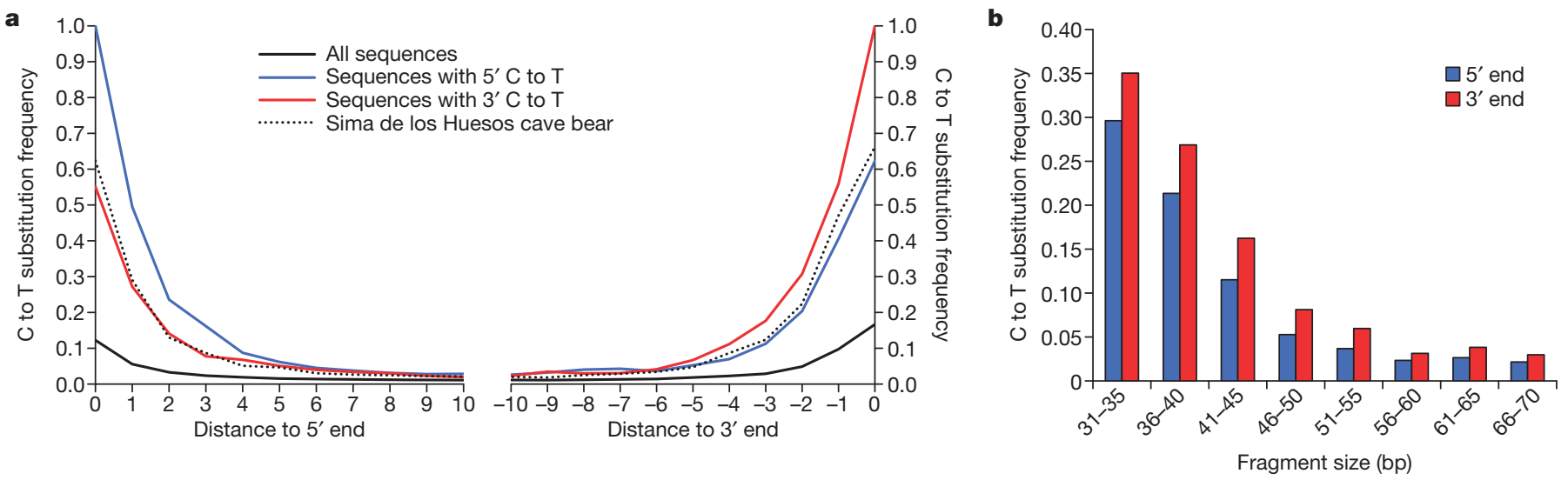

Figure $3 \mid$ Patterns of cytosine deamination in the libraries constructed from the Sima de los Huesos hominin femur. a, C to T substitution frequencies are shown for the terminal positions of the aligned sequences for all sequences (black), those sequences carrying a $\mathrm{C}$ to T substitutions at their 59

sequencing results, the libraries yielding the largest number of mitochondrial sequences exhibited very low terminal $\mathrm{C}$ to $\mathrm{T}$ substitution frequencies (\#3\% and \# 6\% at 59 and 39 ends, respectively; Extended Data Fig. 2) indicating that they are dominated by present-day human contamination. Libraries showing $\mathrm{C}$ to $\mathrm{T}$ substitution frequencies of less than $5 \%$ at either end were considered to be too contaminated and therefore disregarded in subsequent analyses.

Variation in $\mathrm{C}$ to $\mathrm{T}$ substitution frequencies among libraries suggest that two populations of sequences are present in the data, an endogenous population strongly affected by cytosine deamination and a contaminating population showing much less deamination. To test if this is the case, we determined the $59 \mathrm{C}$ to $\mathrm{T}$ substitution frequencies for sequences showing a $39 \mathrm{C}$ to $\mathrm{T}$ difference to the reference and vice versa, thereby enriching for putatively endogenous DNA. C to T substitution frequencies indeed increased to $55 \%$ at 59 ends and $62 \%$ at 39 ends, numbers that are close to those determined for the $U$. deningeri sample from Sima de los Huesos ${ }^{14}$ (Fig. 3a). Furthermore, stratification

of the deamination signal by fragment length shows that the endogenous DNA is primarily present among sequences that are shorter than $45 \mathrm{bp}$, again in agreement with the situation in the $U$. deningeri sample (Fig. $3 \mathrm{~b}$ and Extended Data Fig. 3). Based on these results, we removed sequences longer than $45 \mathrm{bp}$ and those that do not carry a terminal C to T substitution on either the 59 or 39 end (Extended Data Table 3). In addition, we applied a mapping quality filter to ensure unique placement of the sequences within the mtDNA genome and readjusted the alignment parameters to tolerate up to five $C$ to $T$ differences but no more than two other differences to the reference mtDNA sequence to discriminate against spurious alignments. Finally, $T$ bases at the first and last three positions of each sequence were masked to reduce the impact of deamination-induced substitutions during consensus calling.

We first called consensus bases for 15,181 positions of the mitochondrial genome that were covered by 5 or more sequences of which at least $80 \%$ agreed. Average coverage across these positions was 21.8. However, such strict filtering increases the risk of ascertainment bias because residual modern human contamination as well as capture and mapping biases may lead to the exclusion of positions where the Sima de los Huesos specimen differs from the probes or the reference sequence. We therefore built a second more inclusive consensus by considering the three terminal positions while selecting sequences with $\mathrm{C}$ to $\mathrm{T}$ substitution and lowering the requirements for coverage and consensus agreement to 3 and $.67 \%$, respectively. This consensus encompasses 16,302 positions or ,98\% of the human mitochondrial reference gen-ome, with an average coverage of 31.6 (Extended Data Fig. 4). Third, to evaluate whether the use of Denisovan capture probes influence the results, we built a consensus using the strictest filtering criteria described ends (blue), at their 39 ends (red), and for all Sima de los Huesos cave bear sequences from the $U$. deningeri sample ${ }^{9}$ (dotted line). $b$, $\mathrm{C}$ to $\mathrm{T}$ substitution frequencies at the first and last base of sequences in different fragment length bins.

above, but including only sequences isolated with present-day human mtDNA probes (Extended Data Fig. 5).

We reconstructed phylogenetic trees in a Bayesian statistical framework 24 using the three Sima de los Huesos consensus mtDNA sequences as well as the mtDNAs of present-day and ancient humans, Neanderthals, Denisovans, chimpanzees and bonobos. All three trees support a topology in which the Sima de los Huesos mtDNA shares a common ancestor with Denisovan mtDNAs to the exclusion of the other mtDNAs ana-lysed with maximum posterior probability (Fig. 4 and Extended Data Fig. 6). As expected owing to its age, the branch leading to the Sima de los Huesos mtDNA is shorter than those leading to any of the other archaic or present-day humans. Using 13 directly dated ancient mtDNA sequences for calibration 25 and the three consensus sequences, we

esti-mated the age of the Sima de los Huesos specimen based on the length of its mtDNA branch (Table 1). These dates vary between 0.15 to 0.64 million years with point estimates close to 400,000 years. This is in striking agreement with the point estimate of 409,000 years for the $U$. deningeri mtDNA ${ }^{14}$. We similarly estimated the divergence

times of the major mitochondrial lineages (Table 1 and Extended Data Table 4) and find that the estimates for the divergence of the mtDNAs of the

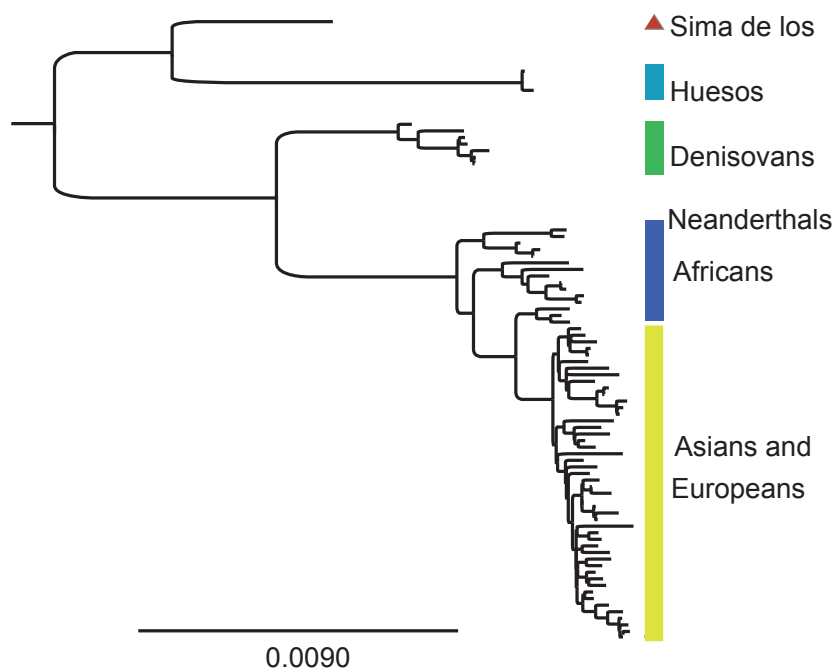

Figure 4 | Bayesian phylogenetic tree of hominin mitochondrial relationships based on the Sima de los Huesos mtDNA sequence determined using the inclusive filtering criteria. All nodes connecting the denoted hominin groups are supported with posterior probability of 1. The tree was rooted using chimpanzee and bonobo mtDNA genomes. The scale bar denotes substitutions per site. 
Table 1 Divergence times of the major hominin mtDNA lineages and the age of the Sima de los Huesos specimen as estimated by using three different filtering strategies for consensus calling

Mitochondrial lineages

Divergence dates and molecular age estimates in Myr BP (95\% highest posterior density (HPD) intervals in brackets)

\begin{tabular}{|c|c|c|c|}
\hline & Strict filters & Inclusive filters & $\begin{array}{l}\text { Strict filters enriched with } \\
\text { human probes only }\end{array}$ \\
\hline Sima de los Huesos age estimate & $0.38(0.18-0.60)$ & $0.37(0.16-0.59)$ & $0.40(0.15-0.64)$ \\
\hline Human - Neanderthal & $0.51(0.35-0.69)$ & $0.53(0.36-0.72)$ & $0.44(0.29-0.61)$ \\
\hline Sima de los Huesos/Denisova - Human/Neanderthal & $0.99(0.70-1.33)$ & $1.04(0.72-1.41)$ & $0.83(0.54-1.11)$ \\
\hline Sima de los Huesos - Denisova & $0.73(0.50-1.01)$ & $0.76(0.51-1.06)$ & $0.65(0.40-0.92)$ \\
\hline
\end{tabular}

Sima de los Huesos hominin and Densiovans vary between 0.40 and 1.06 million years with point estimates around 700,000 years agథhe fact that the Sima de los Huesos mtDNA shares a common ancestor with Denisovan rather than Neanderthal mtDNAs is unex-pected in light of the fact that the Sima de los Huesos fossils carry Neanderthal-derived features (for example, in their dental, mandibular, midfacial, supraorbital and occipital morphology $2,6,7,26$
on DNA sequences retrieved from a manual phalanx and a molar found in southern Siberia9,23. Based on analyses of their nuclear genome 9,10 they are a sister group of Neanderthals, although the mtDNAs of Neanderthals and present-day humans share an mtDNA ancestor more recently with each other than with Denisovans ${ }^{23}$. This may be owing to either incomplete lineage sorting in the common ancestral populations of these groups or to gene flow into Denisovans from another archaic group 9

Several evolutionary scenarios are compatible with the presence of a mtDNA sequence that falls on the Denisovan mtDNA lineage in a ,400,000-year-old hominin in western Europe. First, the Sima de los Huesos hominins may be closely related to the ancestors of Denisovans. However, this seems unlikely, because the presence of Denisovans in west-ern Europe would indicate an extensive spatial overlap with Neanderthal ancestors, raising the question how the two groups could genetically diverge while overlapping in range. Furthermore, although almost no morphological information is available for Denisovans, a molar that carries Denisovan DNA is of exceptionally large size ${ }^{9}$ and does not exhibit the cusp reduction seen in the Sima de los Huesos hominins 7 . Most probably predates the population split time between Denisovans and Neanderthals, which is estimated to one-half to two-thirds of the time to the split between Neanderthals and modern humans, which is esti-mated to be 170,000 to 700,000 years ago ${ }^{9}$. Second, it is possible that the Sima de los Huesos hominins represent a group distinct from both Neanderthals and Denisovans that later perhaps contributed the mtDNA to Denisovans. However, this scenario would imply the independent emergence of several Neanderthal-like morphological features in a group unrelated to Neanderthals. Third, the Sima de los Huesos homi-nins may be related to the population ancestral to both Neanderthals and Denisovans. Considering the age of the Sima de los Huesos remains and their incipient Neanderthal-like morphology, this scenario seems plausible to us, but it requires an explanation for the presence of two deeply divergent mtDNA lineages in the same archaic group, one that later recurred in Denisovans and one that became fixed in Neanderthals, respectively. A forth possible scenario is that gene flow from another hominin population brought the Denisova-like mtDNA into the Sima de los Huesos population or its ancestors. Such a hominin group might have also contributed mtDNA to the Densiovans in Asia 9,10 Based on the fossil record more than one evolutionary lineage may have existed in Europe during the Middle Pleistocene 27 . Several fossils have been found in Europe as well as in Africa and Asia that are close in time to Sima de los Huesos but do not exhibit clear Neanderthal traits. These fossils are often grouped into $\mathrm{H}$. heidelbergensis, a taxon that is difficult to define $8,28,29$, particularly with regard to whether the Sima de los Huesos hominins should be included 8 . Furthermore, there may have been relict populations of still earlier hominins, notably those classified as Homo antecessor, which share some morphological traits with Asian
Homo erectus ${ }^{30}$ and have been found just a few hundred metres away from Sima de los Huesos in Gran Dolina.

Although nuclear sequence data are needed to clarify the genetic relationship of the Sima de los Huesos hominins to Neanderthals and Densiovans, the mtDNA sequence establishes an unexpected link between Denisovans and the western European Middle Pleistocene fossil record. Future efforts will now focus on describing the mtDNA variation of the Sima de los Huesos hominins and retrieving nuclear DNA sequences from them. The latter will be a huge challenge given that almost two grams of bone were required to generate the mtDNA sequence even though several hundred copies of mtDNA exist per cell. Although preservation of DNA for such long periods of time may be favoured by unique preservation conditions in the Sima de los Huesos, the present results show that ancient DNA sequencing techniques have become sensitive enough to warrant further investigation of DNA survival at sites where Middle Pleistocene hominins are found.

\section{METHODS SUMMARY}

A detailed description of the methods used for data generation and analysis is provided in the Methods section.

Online Content Any additional Methods, Extended Data display items and Source Data are available in the online version of the paper; references unique to these sections appear only in the online paper.

Carbonell, E. et al. The first hominin of Europe. Nature 452, 465-469 (2008).

2. Arsuaga, J. L., Martinez, I., Gracia, A. \& Lorenzo, C. The Sima de los Huesos crania (Sierra de Atapuerca, Spain). A comparative study. J. Hum. Evol. 33, 219-281 (1997)

3. Arsuaga, J. L. et al. Size variation in Middle Pleistocene humans. Science 277, 1086-1088 (1997)

4. Bermu' dez de Castro, J. M. \& Nicolas, M. E. Palaeodemography of the AtapuercaSH Middle Pleistocene hominid sample. J. Hum. Evol. 33, 333-355 (1997).

5. Bischoff, J. L. et al. Geology and preliminary dating of the hominid-bearing sedimentary fill of the Sima de los Huesos Chamber, Cueva Mayor of the Sierra de Atapuerca, Burgos, Spain. J. Hum. Evol. 33, 129-154 (1997).

6. Martínez, I. \& Arsuaga, J. L. The temporal bones from Sima de los Huesos Middle Pleistocene site (Sierra de Atapuerca, Spain). A phylogenetic approach. J. Hum. Evol. 33, 283-318 (1997)

7. Martino'n-Torres, M.,Bermudez de Castro, J.M., Gomez-Robles, A., Prado-Simon, L.

\& Arsuaga, J. L. Morphological description and comparison of the dental remains from Atapuerca-Sima de los Huesos site (Spain). J. Hum. Evol. 62, 7-58 (2012).

8. Stringer, C. The status of Homo heidelbergensis (Schoetensack 1908). Evol. Anthropol. 21, 101-107 (2012)

9. Reich, D. et al. Genetic history of an archaic hominin group from Denisova Cave in Siberia. Nature 468, 1053-1060 (2010).

10. Meyer, M. et al. A high-coverage genome sequence from an archaic Denisovan individual. Science 338, 222-226 (2012).

11. Ortega, A. I. et al. Evolution of multilevel caves in the Sierra de Atapuerca (Burgos, Spain) and its relation to human occupation. Geomorphology 196, 122-137 (2013).

12. Arsuaga, J. L. et al. Sima de los Huesos (Sierra de Atapuerca, Spain). The site. J. Hum. Evol. 33, 109-127 (1997).

13. Valdiosera, C. et al. Typing single polymorphic nucleotides in mitochondrial DNA as a way to access Middle Pleistocene DNA. Biol. Lett. 2, 601-603 (2006).

14. Dabney, J. et al. Complete mitochondrial genome sequence of a Middle Pleistocene cave bear reconstructed from ultrashort DNA fragments. Proc. Natl Acad. Sci. USA 110, 15758-15763 (2013).

15. Willerslev, E. et al. Ancient biomolecules from deep ice cores reveal a forested southern Greenland. Science 317, 111-114 (2007).

16. Orlando, L. et al. Recalibrating Equus evolution using the genome sequence of an early Middle Pleistocene horse. Nature 499, 74-78 (2013). 
17. Carretero, J. M. et al. Stature estimation from complete long bones in the Middle Pleistocene humans from the Sima de los Huesos, Sierra de Atapuerca (Spain). J. Hum. Evol. 62, 242 255 (2012).

18. Gansauge, M. T. \& Meyer, M. Single-stranded DNA library preparation for the sequencing of ancient or damaged DNA. Nature Protocols 8, 737-748 (2013).

19. Li, H. \& Durbin, R. Fast and accurate short read alignment with Burrows-Wheeler transform. Bioinformatics 25, 1754-1760 (2009).

20. Briggs, A. W. et al. Patterns of damage in genomic DNA sequences from a Neandertal. Proc. Natl Acad. Sci. USA 104, 14616-14621 (2007).

21. Krause, J. et al. A complete mtDNA genome of an early modern human from Kostenki, Russia. Curr. Biol. 20, 231-236 (2010).

22. Sawyer, S., Krause, J., Guschanski, K., Savolainen, V. \& Paabo, S. Temporal patterns of nucleotide misincorporations and DNA fragmentation in ancient DNA. PLOS ONE 7, e34131 (2012).

23. Krause, J. et al. The complete mitochondrial DNA genome of an unknown hominin from southern Siberia. Nature 464, 894-897 (2010).

24. Ronquist, F. \& Huelsenbeck, J. P. MrBayes 3: Bayesian phylogenetic inference under mixed models. Bioinformatics 19, 1572-1574 (2003).

25. Shapiro, B. et al. A Bayesian phylogenetic method to estimate unknown sequence ages. Mol. Biol. Evol. 28, 879-887 (2011).

26. Arsuaga, J. L., Martinez, I., Gracia, A., Carretero, J. M. \& Carbonell, E. Three new human skulls from the Sima de los Huesos Middle Pleistocene site in Sierra de Atapuerca, Spain. Nature 362, 534-537 (1993).

27. Arsuaga, J. L. Colloquium paper: terrestrial apes and phylogenetic trees. Proc. Natl Acad. Sci. USA 107 (Suppl. 2), 8910-8917 (2010).

28. Hublin, J. J. Out of Africa: Modern human origins special feature: The origin of Neandertals. Proc. Natl Acad. Sci. USA 106, 16022-16027 (2009).

29. Mounier, A., Marchal, F. \& Condemi, S. Is Homo heidelbergensis a distinct species? New insight on the Mauer mandible. J. Hum. Evol. 56, 219-246 (2009).
30. Carbonell, E. et al. An Early Pleistocene hominin mandible from Atapuerca-TD6, Spain. Proc. Natl Acad. Sci. USA 102, 5674-5678 (2005).

Acknowledgements We thank J. Dabney, M. Dannemann, C. de Filippo, S. Lippold, K. Pru“fer, M. Slatkin, M. Stiller, C. Valdiosera and B. Viola for discussions and comments on the manuscript; G. Renaud and U. Stenzel for help with sequence data processing; B. Ho"ber and A. Weihmann for performing the sequencing runs; M. Gansauge

P. Korlevic', R. Rodri'guez and I. Uren ₹ for help in the laboratory; M. Schreiber for help with graphics; J. Trueba for providing the fossil image; M. Cruz Ortega for restoration of the fossil and the rest of the members of the Sima de los Huesos excavation team

?for decades of continuous efforts. Genetics work was funded by the Max Planck

?Society and its Presidential Innovation Fund. Field work at the Sierra de Atapuerca sites is funded by the Junta de Castilla y Leo'n and the Fundacio'n Atapuerca.

?Research was supported by Spanish Ministerio de Ciencia e Innovacio'n (project

CGL2009-12703-C03) and Spanish Ministerio de Econom'ia y Competitividad (project CGL2012-38434-C03)

Author Contributions M.M. designed the experiments and analysed the data; Q.F. performed phylogenetic analyses; A.A., I.G. and B.N. performed the experiments; J.-L.A., I.M., A.G., J.M.B. and E.C. excavated the fossil and provided expert archaeological and anthropological information; J.L.A. and S.P. were involved in study design; and M.M., J.-L.A. and S.P. wrote the manuscript.

Author Information The Sima de los Huesos mtDNA consensus sequence (based on the inclusive filtering criteria) is deposited in GenBank under accession number KF683087. Reprints and permissions information is available at www.nature.com/reprints. The authors declare no competing financial interests. Readers are welcome to comment on the online version of the paper. Correspondence and requests for materials should be addressed to M.M. (mmeyer@eva.mpg.de). 


\section{METHODS}

Description of the femur, archaeological context and sampling. The largest femoral fragment (AT-999) was discovered in 1994 and represents the distal (lower) two thirds of the bone. The proximal (upper) third of the femur (AT-2943) was recovered in 1999. The third femoral fragment (AT-2944), also found in 1999, is a much smaller shaft fragment, which partially connects the two larger femoral fragments (see Fig. 2). All three fragments were found close to each other in square U-15 (the Sima de los Huesos excavation grid has squares of $0.5 \mathrm{~m}$ in length). U- 15 excavation square is in the central area of the site and is particularly rich in human fossils, including complete skulls.

Sampling was performed by drilling small holes into the cortical tissue of all three bone fragments starting from pre-existing fractures. To reduce the impact of modern human contamination, approximately $1 \mathrm{~mm}$ of surface material was removed before drilling holes in the bone at low speed (1,000 r.p.m.) using a sterile dentistry drill. No damage was done to the outer surface of the femur.

DNA extraction, library preparation and shotgun sequencing. Using $1.95 \mathrm{~g}$ of bone material in total, 39 DNA extracts were made from between 25 and $75 \mathrm{mg}$ of bone powder each using a recently published silica-based DNA extraction pro-tocol optimized for the recovery of very short molecules from ancient biological material ${ }^{14}$. Two of these extracts were made from surface material, whereas all other extracts were generated from bone powder sampled from inside the bone. Extraction blank controls were carried alongside with the samples in each set of DNA extractions. As substantial pellets of undigested bone powder remained after extraction, we also generated re-extracts from 6 bone pellets to investigate whether additional DNA can be released by repeating the extraction. Libraries were prepared in sets of 16 using between 20 and $30 \mu \mathrm{l}$ of sample or blank DNA extract (out of $50 \mu \mathrm{l}$ total volume) and following a single-stranded library preparation protocol

specifically developed for highly degraded ancient DNA 18 . One positive control DNA glycosylase treatment was performed to preserve the $\mathrm{C}$ to $\mathrm{T}$ substitution patterns that are typical for sequences from ancient $\mathrm{DNA}^{20}$.

The number of unique molecules in each library was estimated by quantitative PCR (qPCR), using primers hybridizing to the adaptor sequences ${ }^{18}$. All sample libraries $(n=77)$ yielded $\mathrm{QPCR}$ molecule counts between $1.9 \times 10^{9}$ and $2.4 \times 10^{10}$, with exception of the libraries prepared from re-extracted bone pellets, which returned numbers in the range of $1.3 \times 10^{8}$ to $1.1 \times 10^{9}$ (Extended Data Table 1$)$.
Molecule counts of the extraction and library preparation blanks $(n=13)$, which represent artefacts and library molecules derived from contamination with exo-

genous DNA, were consistently lower (in the range of $4.9 \times 10^{6}$ to $4.8 \times 10^{7}$ ).
qPCR molecule counts thus indicate that substantial amounts of DNA reside in the bone and that most of this DNA is successfully released in a single round of DNA extraction.

Each library was divided into four aliquots of $12.5 \mu \mathrm{l}$, which were then amplified into PCR plateau in $100 \mu \mathrm{l}$ reactions with AccuPrime Pfx DNA polymerase (Life Technologies) as described elsewhere ${ }^{31}$. During amplification, two unique index sequences were introduced into the adaptors of each library, following a doubleindexing scheme described elsewhere 32 . Amplification products from the same library were pooled and purified using the MinElute PCR purification kit (Qiagen).

Aliquots from a subset of libraries were pooled and subjected to shallow shotgun sequencing using Illumina's MiSeq platform in double-index configuration $(2 \times 76+$ $2 \times 7$ cycles) ${ }^{32}$. Raw sequence data were processed as described below. The fragment size distribution of the sequences before mapping shows a mode around 30 bp (Extended Data Fig. 1), indicating that small DNA fragments were efficiently extracted and converted into library molecules. Sequences were aligned against the human genome (GRCh37/1000 Genomes release) using BWA ${ }^{19}$ with relaxed alignment parameters 10 . In most libraries, less than $0.1 \%$ of the sequences $\geq 35 \mathrm{bp}$ mapped against the human genome with a mapping quality $\geq 30$ (see Extended Data Table 2). Owing to the small numbers of aligned sequences, $C$ to $T$ substitution frequencies cannot be determined with confidence in most cases. A small number of libraries, most notably the ones generated from surface material, stand out with high fractions of aligned sequences (up to $8.4 \%$ ). However, the $\mathrm{C}$ to $\mathrm{T}$ substitution frequencies in these libraries are extremely low, indicating that the vast majority of sequences are derived from modern human contamination. Based on these analyses, none of the libraries prepared from the femur is a suitable candidate for deeper shotgun sequencing.

Enrichment of mitochondrial DNA and sequencing. All sample and blank libraries were first enriched for mitochondrial DNA using present-day human mitochondrial probes synthesized on an oligonucleotide array (in 3-bp tiling den-sity, using human mtDNA sequence NC_001807), following the method described in ref. 33 , except that the hybridization and wash temperatures were lowered to $60^{\circ} \mathrm{C}$ and $55^{\circ} \mathrm{C}$ to facilitate enrichment of short library molecules ${ }^{14}$. After phylo-genetic analyses showed that the mitochondrial genome of the Sima de los Huesos hominin is closer to Denisovans than modern humans, the libraries were also enriched using Denisovan mitochondrial probes. To construct these probes, 19 overlapping DNA fragments of approximately $1 \mathrm{~kb}$ were designed (GeneArt Fragments, Life Technologies) using the mitochondrial genome of the Denisovan manual phalanx 23 as reference. The fragments encompassed the following sequence coordinates: $319-1289$, 1223-2191, 2101-3088, 3018-3950, 3897-4889, 4806$5763, \quad 5688-6663,6612-7601, \quad 7529-8486, \quad 8428-9418, \quad 9371-10300, \quad 10203-$ $11156,11085-12017,11966-12931,12881-13813,13762-14706,14641-15600$, 15551-16503 and 16460-381. One of the fragments (8428-9418) failed several synthesis attempts and could not be included in the probe pool. The 18 successfully synthesized fragments were amplified with Q5 Hot Start High-Fidelity DNA Polymerase (NEB) according to the supplier's instructions using a $5^{\prime}$ biotinylated forward primer and an unmodified reverse primer. Amplified fragments were purified using solid-phase reversible immobilization (SPRI) beads as described elsewhere $e^{34}$ and pooled in equimolar ratios. Bead capture was performed as described in ref. 35, but with lowered hybridization and wash temperatures as detailed above. Two successive rounds of hybridization enrichment were carried out with both probe sets.

The enriched libraries were combined into three pools and sequenced on Illumina's HiSeq 2500 platform in rapid mode, using recipes for paired-end sequencing with two index reads $(96+7+96+7 \text { o r } 76+7+76+7 \text { cycles })^{32}$. The first pool (including libraries B2949-B2994 enriched with present-day human probes) was sequenced together with libraries from another experiment (mitochondrial captures of ancient human samples), occupying $75 \%$ of two lanes of a flow cell. The second and third pools (including libraries A1543-A2045 enriched with present-day human probes and all libraries enriched with Denisovan probes, respectively) were sequenced on one lane of a flow cell each.

Raw data processing and mapping. Base calling was performed using Bustard (Illumina) or freeIbis ${ }^{36}$. Sequences that did not perfectly match one of the expected
index combinations were discarded and full-length molecule sequences were reconstructed by overlap-merging of paired-end reads 37 . Merged sequences $\geq 30 \mathrm{bp}$ were aligned against the revised Cambridge reference sequence (NC_012920) using BWA ${ }^{19}$ with the parameters ' $-\mathrm{n} 5$ ', which allows up to five mismatches, and '-1 16500', which turns off seeding. Sequences with identical alignment start and end coordinates were collapsed into single sequences by consensus calling 23 . Enrichment success and recovery of mitochondrial sequences. The efficiency of enrichment varied between the human and Denisovan probe sets, with $6.8 \%$ and $27.6 \%$ of the sequences $\geq 30 \mathrm{bp}$ aligning to the human mitochondrial reference genome before duplicate removal (compare Extended Data Table 3). Each unique sequence is represented by 21 duplicates on average (but note that this value is deflated by few libraries yielding very large numbers of sequences; see Extended Data Table 1), indicating that the libraries were sequenced to exhaustion. There are remarkable differences in the number of unique sequences obtained from each library (Extended Data Table 1 and Extended Data Fig. 2), ranging from 122 to 719 for the blank controls, from 448 to 9,757 for the libraries prepared from reextracted bone pellets, and from 1,529 to 773,319 for the regular sample libraries. Extended Data Table 3 summarizes the number of sequences retained in the sample libraries after each processing step.

In previous work on the cave bear sample from Sima de los Huesos, almost all sequenced fragments $(94 \%)$ were $\leq 50 \mathrm{bp}$ in length ${ }^{14}$. The fragment size distribution inferred from the hominin sequences exhibits a larger proportion of longer molecules (Extended Data Fig. 3), possibly reflecting contaminant sequences. When stratifying terminal $\mathrm{C}$ to $\mathrm{T}$ substitution frequencies by sequence length, we find a strong decline of the deamination signal with length (Fig. 3b), indicating that the pronounced tail of long sequences is due to modern human contamination. Basic filters applied to the sequences before consensus calling. We used the following set of filters to decrease the load of modern human contamination and to eliminate spurious alignments before consensus calling. The number of sequences retained after each step of filtering is provided in Extended Data Table 3. First, we excluded libraries without substantial signals of cytosine deamination $(<5.0 \%$ terminal C to $\mathrm{T}$ substitution frequencies). Second, for mapping the sequences with BWA we allowed up to 5 mismatches and one insertion or deletion to prevent the loss of sequences with several damage-derived $\mathrm{C}$ to $\mathrm{T}$ substitutions, but these parameters are extremely permissive and do not sufficiently discriminative against spurious alignments. We therefore removed sequences showing more than two differences to the human mitochondrial reference genome that cannot be explained by cytosine deamination (that is, sequences with more than two non-C-to-T sub-stitutions in the orientation as sequenced). In addition, we limited the maximum number of acceptable differences to the reference to five, counting also insertions or deletions. Third, sequences with a mapping quality of less than 30 were removed to ensure secure placement within the mitochondrial genome. Fourth, sequences longer than $45 \mathrm{bp}$ were removed, because they are particularly rich in contamina-tion (see Fig. 3b). 
Overview of the consensus calling procedure. As the patterns of cytosine deamination showed that all libraries are substantially contaminated with modern human DNA, we used terminal $\mathrm{C}$ to $\mathrm{T}$ substitutions to enrich for endogenous sequences before consensus calling. The simplest approach for identifying sequences with deamination-induced $\mathrm{C}$ to $\mathrm{T}$ substitutions is by comparison to the human mitochondrial reference genome. However, human contaminants will occasion-ally show true $\mathrm{C}$ to $\mathrm{T}$ differences to the reference due to sequence divergence. To reduce carryover of such sequences, we developed another approach where we isolated sequences with a terminal $\mathrm{T}$ (or a $\mathrm{T}$ in the first three or last three positions; see below) if $80 \%$ or more of all sequences covering the respective position in the mitochondrial genome show a $\mathrm{C}$. This procedure accounts for the fact that a $\mathrm{C}$ to $\mathrm{T}$ change is not indicative of cytosine deamination if it is shared by many other sequences, irrespective of the state of the reference. Information about the state of all sequences at each position of the mitochondrial genome was obtained using the 'mpileup' command implemented in SAMtools ${ }^{38}$.

To reduce the effect of damage-induced $\mathrm{C}$ to $\mathrm{T}$ substitutions during consensus calling, we next converted Ts to Ns in the first and last three positions of each sequence. In these positions, cytosines are converted to uracils with frequencies $\gg 10 \%$ (see Fig. 3a). We again took the state of all other sequences into account, only converting $\mathrm{T}$ to $\mathrm{N}$ if at least one other sequence showed a $\mathrm{C}$ at the respective position.

After masking terminal $\mathrm{C}$ to $\mathrm{T}$ substitutions, the 'mpileup' command was used again to convert the BAM alignment file into a position-based tabular format. This table was used to determine (1) the coverage at each position, (2) the consensus base (based on a simple majority vote), and (3) the percentage of sequences supporting the majority base ('consensus support'). Ns were disregarded in all three measures. As BWA does not account for the circularity of the mitochondrial genome, mapping, filtering and consensus calling were repeated using a modified reference sequence where $1 \mathrm{~kb}$ of sequence was moved from start to end. This way we obtained the same measures also for the first and last bases of the mitochondrial genome.

Constructing consensus sequences under different filtering regimes. Accurate reconstruction of the mitochondrial genome sequence of the hominin femur sample is complicated by the high background of modern human contamination and the short size of endogenous DNA fragments. Short fragments are less effi-ciently enriched in hybridization capture ${ }^{14}$, even more so if their sequences show differences to the capture probe. In addition, mapping bias may reduce the probability of identifying endogenous sequences if they differ from the reference sequence. Both mapping bias and contamination, if not effectively removed, would make the hominin consensus sequence more similar to modern human mitochon-drial DNA. Capture bias goes in the same direction for the sequences enriched with presentday human probes, but is expected to increase similarity with Denisovans when using Denisovan probes. As the effects of modern human contamination, capture bias and mapping bias are expected to increase with the stringency of filtering (that is, with more stringent cutoffs for coverage and consensus support), we reconstructed the consensus sequence using three different filtering strategies to test whether filtering influences the results of the phylogenetic analyses.

The first consensus sequence is based on a very strict filtering regime and includes the positions that can be determined with highest confidence. Sequences were filtered for $\mathrm{C}$ to $\mathrm{T}$ substitutions based on the first or last base of each sequence only, thus using the positions providing most power to discriminate between contaminant and endogenous sequences. We then required a minimum coverage of 5 and a consensus support $>=80 \%$ in order to call a consensus base. After visual inspection of the sequence alignments we removed three stretches of C-rich homopolymer sequence from the consensus (positions 286-315, 956-965 and 16180-16193 according to the revised Cambridge reference sequence (rCRS) coordinate system), because they are difficult to resolve with sequences enriched for $\mathrm{C}$ to $\mathrm{T}$ substitutions. With this procedure, $15,181 \mathrm{bp}$ of the mitochondrial genome ( $\sim 92 \%$ of the reference sequence) could be determined. Each determined position is covered 21.8 times on average. Coverage distribution along the mito-chondrial genome is provided in Extended Data Fig. 4.

The second consensus sequence is more inclusive and recovers a larger fraction of the mitochondrial genome. We filtered the sequences for $\mathrm{C}$ to $\mathrm{T}$ substitutions using the first and last three bases of each sequence, thereby increasing the number of sequences available for consensus calling from 10,160 to 15,528 (Extended Data Table 3). In addition, we lowered the threshold for consensus calling to a min-imum coverage of 3 and the required consensus support to be $>67 \%$. Three C-rich homopolymer stretches were removed as described above. Using this less stringent approach, the number of determined bases increases to $16,302(\sim 98 \%$ of the mitochondrial genome) and average coverage is 31.6 for the positions that were determined (compare Extended Data Fig. 4).

The third consensus sequence was generated to test whether phylogenetic analyses are influenced by the use of Denisovan capture probes. For this purpose we reprocessed the sequence data from the start, using only sequences generated in capture experiments with present-day human probes. A consensus was then called using the high-confidence criteria described above, yielding base calls for 13,157 positions of the mitochondrial genome determined with 16.3 -fold average coverage. The sequence coverage of the mitochondrial genome obtained from the enrichments with present-day human probes (and for comparison with Denisovan probes) is shown in Extended Data Fig. 5.

Phylogenetic reconstructions. Multiple sequence alignments were generated separately for each of the Sima de los Huesos consensus sequences using complete mitochondrial genome sequences of a worldwide panel of 54 present-day humans ${ }^{39}, 9$ ancient humans ${ }^{40}, 7$ Neanderthals ( 6 described in literature 41 and one deposited in GenBank with accession number KC879692), 2 Denosivans $9,23,22$ bonobos ${ }^{42}$ and 24 chimpanzees 43 using MAFFT 44 . After removing the D-loop (rCRS positions 16023-577), we selected the general time reversible substitution model with invari-ant sites and a gamma distributed correction for rate heterogeneity $(G T R+I+\Gamma)$ as suggested by MODELTEST ${ }^{45}$. Phylogenetic trees were reconstructed in a Bayesian statistical framework using MrBayes 24 . We performed four independent runs of Markov Chain Monte Carlo (MCMC) sampling with 30,000,000 generations, respectively. In each run, the first 3,000,000 generations were discarded as burn-in. All four consensus trees show the same topology of the major mitochondrial lineages (Fig. 4 and Extended Data Fig. 6) and group the Sima de los Huesos sequence with Denisovans with a posterior probability of 1 .

We further estimated the divergence times among major mitochondrial lineages as well as the age of the Sima de los Huesos mitochondrial sequence based on its branch length in a Bayesian statistical framework 25 as implemented in BEAST 46 . For this analysis we used the same data set as described above in the MrBayes section. To inform the molecular clock rate estimate we used nine ancient modern human and four Neanderthal complete mitochondrial genome sequences from radiocarbon dated specimens ${ }^{40}$. For the ancient individuals of unknown age we used uniform priors ranging from 0 to $1,000,000$ years BP. Two different models of rate variation among branches were tested: a strict clock and an uncorrelated lognormaldistributed relaxed clock, both under a constant size and a Bayesian skyline coalescent tree prior. For each of these four analyses, two Markov chain Monte Carlo (MCMC) runs of 30,000,000 generations with samples taken every 1,000 generations were performed, respectively. The first 6,000,000 iterations were discarded as burn-in and the remaining were combined using LogCombiner, resulting in a total of 48,000,000 generations per analysis to ensure sufficient sampling of parameters with effective sample sizes (ESS) of $>200$. When compar-ing the strict versus the relaxed clock model using Bayes Factors test 47 , we found strong support in favour of the relaxed clock model $(\log 10 \mathrm{BF} \geq 1.13$ for all three consensus sequences). The constant size coalescent could not be rejected over the Bayesian skyline coalescent $\left(\log _{10} \mathrm{BF} \leq 0.39\right.$ for all three consensus sequences). We therefore used the analysis based on the relaxed clock model and the constant size coalescent before proceed estimating the divergence times among various clades as reported in Table 1 and Extended Data Table 4.

The divergence time estimates are stable and independent of which Sima de los Huesos consensus sequence was used (see the consensus calling section above). The 95\% highest posterior density (HPD) intervals of all estimates are in agree-ment and include previous estimates based on mtDNA sequences, for example, for the divergence between humans and chimpanzees 4.2-5.2 $\mathrm{Myr}$ ago $^{48}$, between Denisovans and modern humans $0.6-1.3 \mathrm{Myr}$ ago ${ }^{23,40}$, between Neanderthal and modern humans $0.3-0.6 \mathrm{Myr}$ ago ${ }^{41,49}$, and between chimpanzees and bonobos $1.5-$ $2.1 \mathrm{Myr}$ ago ${ }^{50}$ as well as the time to the most recent common ancestor of all humans around $120,000-236,000$ years ago 40,51

31. Dabney, J. \& Meyer, M. Length and GC-biases during sequencing library amplification: a comparison of various polymerase-buffer systems with ancient and modern DNA sequencing libraries. Biotechniques 52, 87-94

32. Kiocter, M., Sawyer, S. \& Meyer, M. Double indexing overcomes inaccuracies in multiplex sequencing on the Illumina platform. Nucleic Acids Res. 40, e3 (2012).

33. Fu, Q. et al. DNA analysis of an early modern human from Tianyuan Cave, China. Proc. Natl Acad. Sci. USA 110, 2223-2227 (2013).

34. Rohland, N. \& Reich, D. Cost-effective, high-throughput DNA sequencing libraries for multiplexed target capture. Genome Res. 22, 939-946 (2012).

35. Maricic, T., Whitten, M. \& Paabo, S. Multiplexed DNA sequence capture of mitochondrial genomes using PCR products. PLOS ONE 5, e14004 (2010).

36. Renaud, G., Kircher, M., Stenzel, U. \& Kelso, J. freelbis: an efficient basecaller with calibrated quality scores for Illumina sequencers. Bioinformatics $\mathbf{2 9}$ 1208-1209 (2013)

37. Kircher, M. Analysis of high-throughput ancient DNA sequencing data. Methods Mol. Biol. 840, 197-228 (2012).

38. Li, H. et al. The Sequence Alignment/Map format and SAMtools. Bioinformatics 25, 2078-2079 (2009).

39. Ingman, M., Kaessmann, H., Paabo, S. \& Gyllensten, U. Mitochondrial genome variation and the origin of modern humans. Nature 408, 708-713 (2000).

40. Fu, Q. et al. A revised timescale for human evolution based on ancient mitochondrial genomes. Curr. Biol. 23, 553-559 (2013). 
41. Briggs, A. W. et al. Targeted retrieval and analysis of five Neandertal mtDNA genomes. Science 325, 318-321 (2009).

42. Zsurka, G. et al. Distinct patterns of mitochondrial genome diversity in bonobos (Pan paniscus) and humans. BMC Evol. Biol. 10, 270 (2010).

43. Bjork, A., Liu, W., Wertheim, J. O., Hahn, B. H. \& Worobey, M. Evolutionary history of chimpanzees inferred from complete mitochondrial genomes. Mol. Biol. Evol. 28, 615-623 (2011).

44. Katoh, K. \& Standley, D. M. MAFFT multiple sequence alignment software version 7: improvements in performance and usability. Mol. Biol. Evol. 30, 772-780 (2013).

45. Posada, D. \& Crandall, K. A. MODELTEST: testing the model of DNA substitution. Bioinformatics 14, 817-818 (1998).
46. Drummond, A. J. \& Rambaut, A. BEAST: Bayesian evolutionary analysis by sampling trees. BMC Evol. Biol. 7, 214 (2007).

47. Kass, R. E. \& Raftery, A. E. Bayes Factors. J. Am. Stat. Assoc. 90, 773-795 (1995).

48. Horai, S. et al. Man's place in Hominoidea revealed by mitochondrial DNA genealogy. J. Mol. Evol. 35, 32-43 (1992).

49. Green, R. E. et al. A complete Neandertal mitochondrial genome sequence determined by high-throughput sequencing. Cell 134, 416-426 (2008).

50. Stone, A. C. etal. More reliable estimates of divergence times in Pan using complete mtDNA sequences and accounting for population structure. Phil. Trans. R. Soc Lond. B 365, 3277-3288 (2010).

51. Soares, P. et al. Correcting for purifying selection: an improved human mitochondrial molecular clock. Am. J. Hum. Genet. 84, 740-759 (2009). 


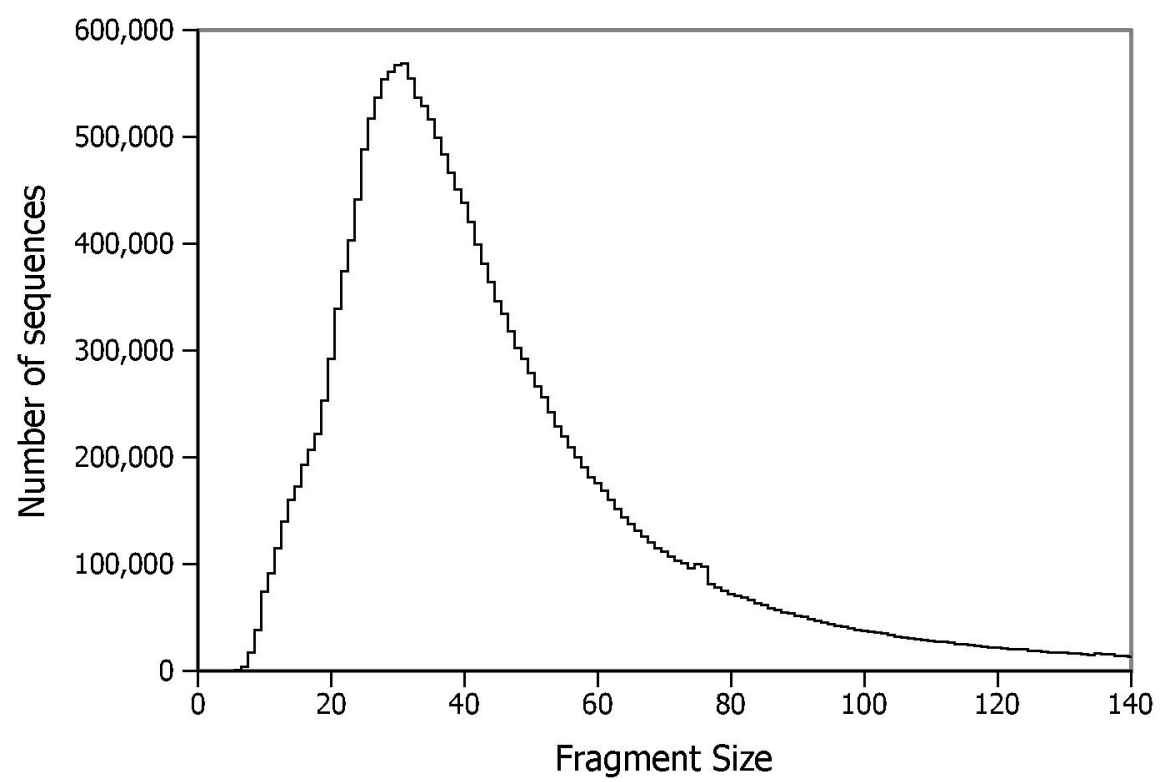

Extended Data Figure 1 | Size distribution of all overlap-merged sequences generated by shotgun sequencing (before mapping). 


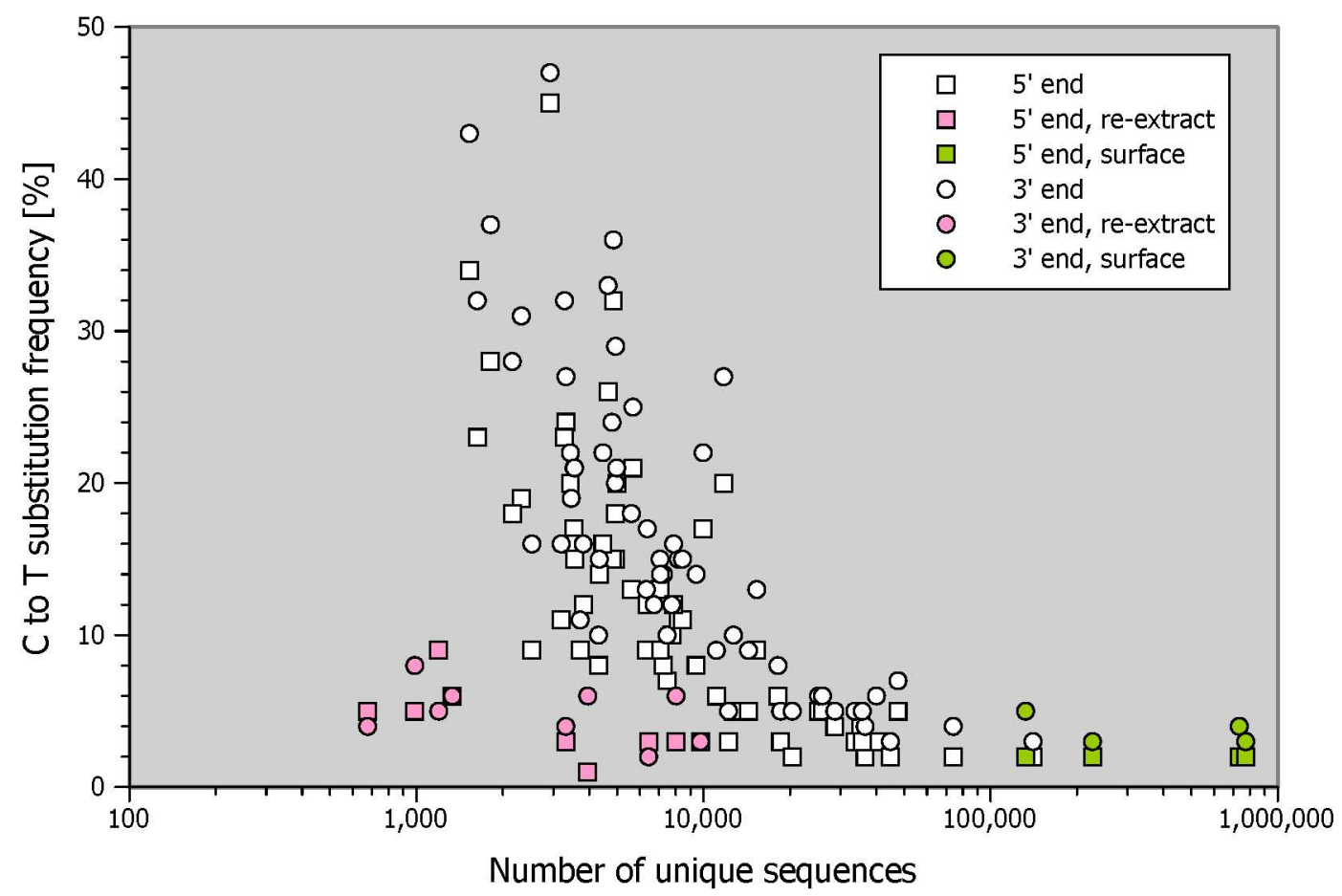

Extended Data Figure $2 \mid 59$ and $39 \mathrm{C}$ to T substitution frequencies plotted against the number of unique mitochondrial sequences retrieved from each sample library. Libraries prepared from re-extracted pellets or surface material are highlighted in colour. 


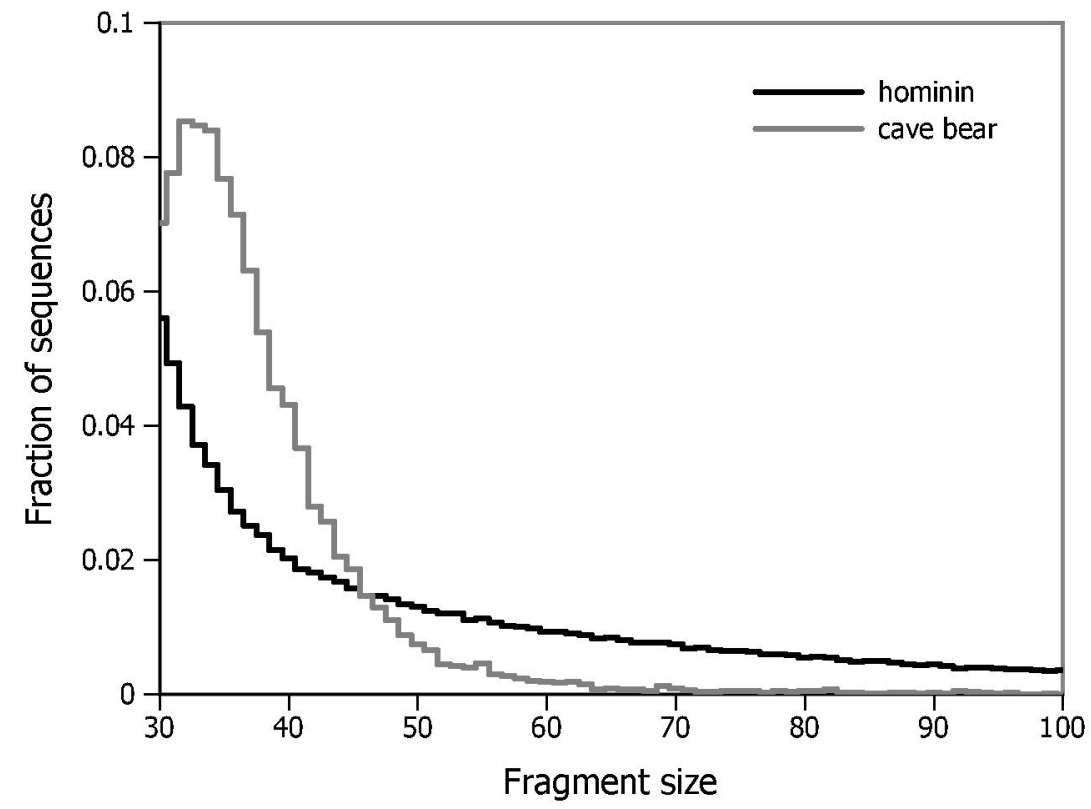

Extended Data Figure $3 \mid$ Sequence length distribution of unique sequences. The distribution obtained from the Sima de los Huesos cave bear is shown for comparison. 


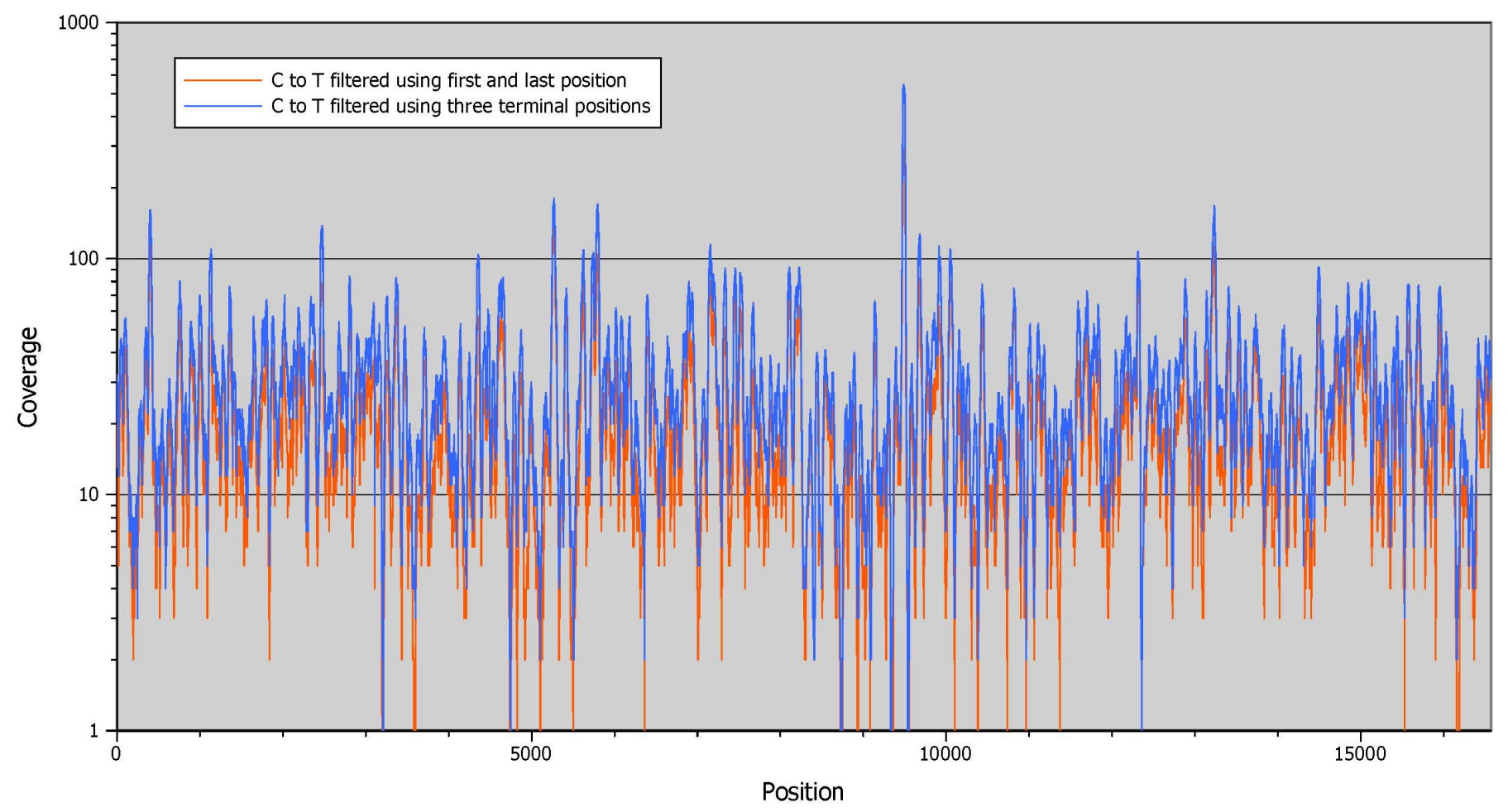

Extended Data Figure $4 \mid$ Sequence coverage of the mitochondrial genome obtained from sequences with terminal $\mathrm{C}$ to $\mathrm{T}$ substitutions. 


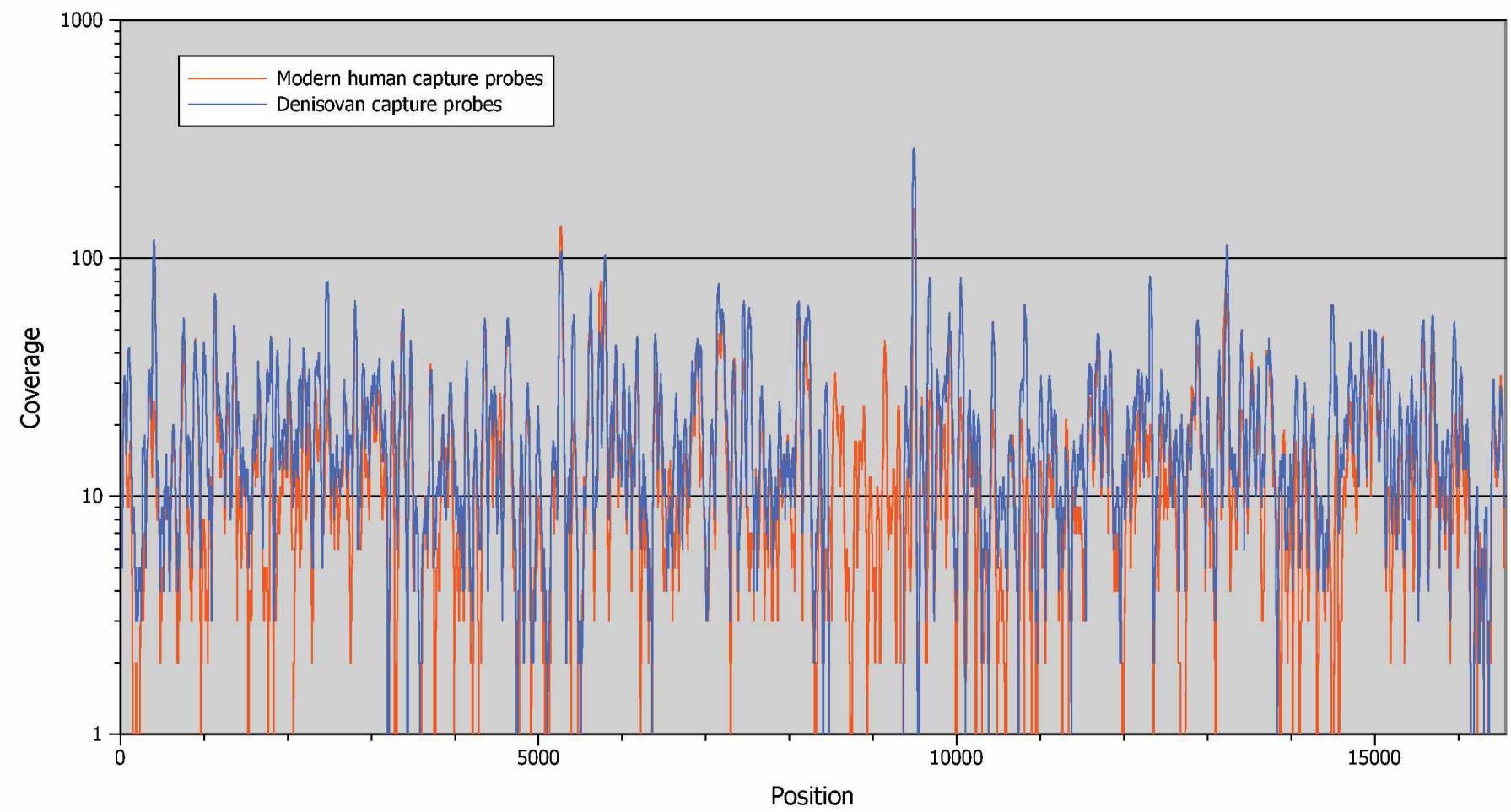

Extended Data Figure 5 | Sequence coverage of the mitochondrial genome plotted separately for both capture probe sets used (based on sequences with a $\mathrm{C}$ to $\mathrm{T}$ substitution at the first or last alignment position). 


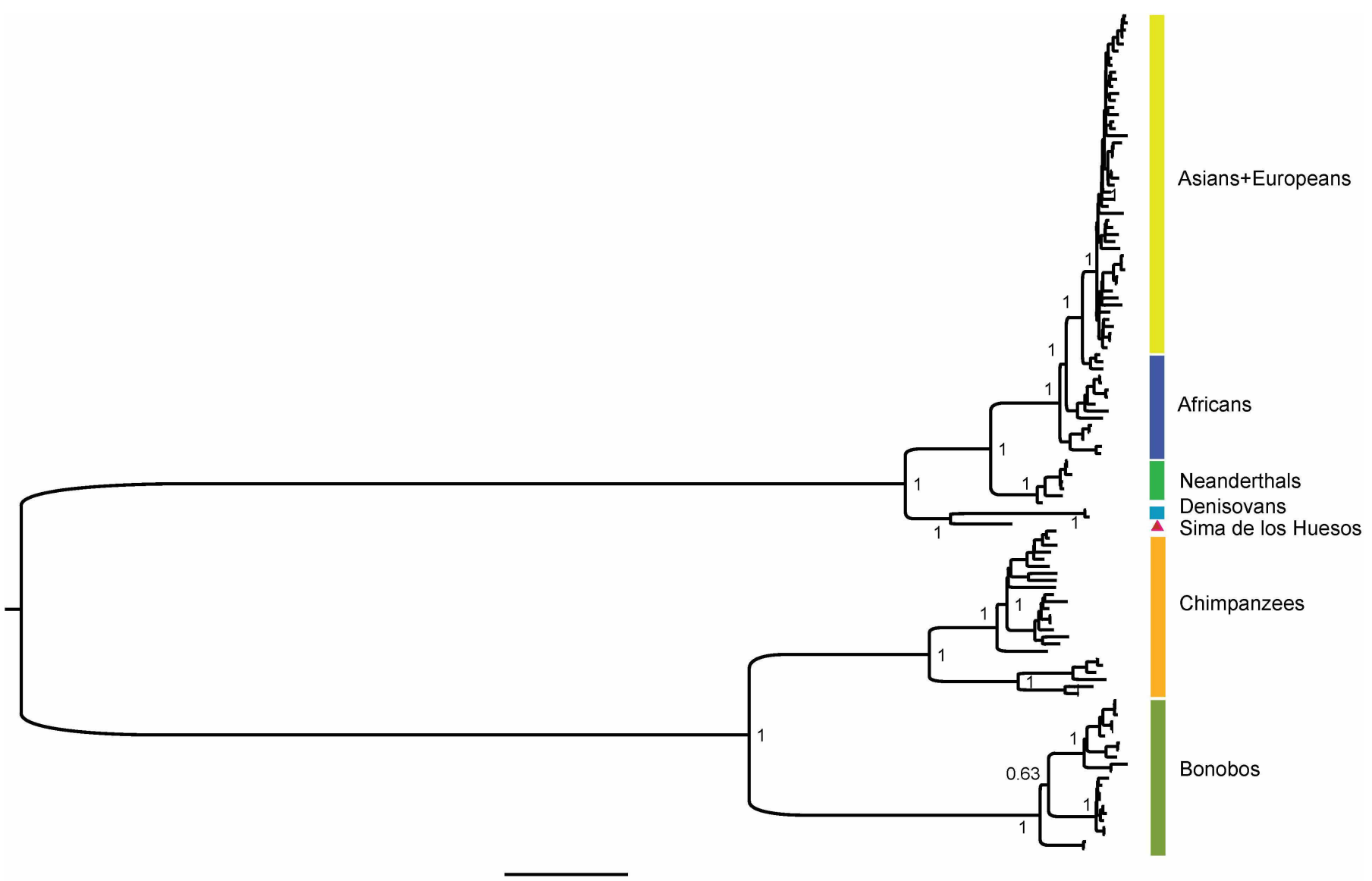

0.0090

Extended Data Figure $6 \mid$ Complete view of the mid-point rooted phylogenetic tree constructed with a Bayesian approach under a GTR 1 I $1 \mathrm{C}$ model of sequence evolution using the Sima de los Huesos

consensus sequence generated with inclusive filters as well as 54 present-day humans, 9 ancient humans, 7 Neanderthals, 2 Denosivans, 22 bonobos and 24 chimpanzees. The posterior probabilities are provided for the major nodes. 
Extended Data Table $1 \mid$ Characteristics of all libraries prepared for this study

\begin{tabular}{|c|c|c|c|c|c|c|c|}
\hline Library set & $\begin{array}{l}\text { Extracted } \\
\text { material }\end{array}$ & $\begin{array}{c}\text { DNA } \\
\text { extract ID }\end{array}$ & $\begin{array}{c}\text { Bone } \\
\text { material } \\
\text { used for } \\
\text { DNA extr. } \\
\text { [mg] }\end{array}$ & Library ID & $\begin{array}{c}\text { Fraction of } \\
\text { extract used } \\
\text { for library } \\
\text { prep. }\end{array}$ & $\begin{array}{c}\mathrm{qPCR} \\
\text { molecule } \\
\text { count }\end{array}$ & $\begin{array}{c}\text { Unique } \\
\text { mtDNA } \\
\text { sequences } \\
(>=30 \mathrm{bp})\end{array}$ \\
\hline 1 & sample & E1385 & 25 & B2949 & 0.4 & $1.40 \mathrm{E}+10$ & 3,717 \\
\hline 1 & sample & E1386 & 33 & B2950 & 0.4 & $1.36 \mathrm{E}+10$ & 4,307 \\
\hline 1 & sample & E1387 & 39 & B2951 & 0.4 & $1.33 E+10$ & 7,754 \\
\hline 1 & sample & E1388 & 47 & B2952 & 0.5 & $1.60 \mathrm{E}+10$ & 3,317 \\
\hline 1 & sample & E1389 & 48 & B2953 & 0.5 & $2.15 \mathrm{E}+10$ & 4,936 \\
\hline 1 & sample & E1390 & 65 & B2954 & 0.4 & $1.91 \mathrm{E}+10$ & 3,280 \\
\hline 1 & blank & E1391 & & B2955 & 0.4 & $3.58 \mathrm{E}+07$ & 433 \\
\hline 1 & sample & E1392 & 42 & B2956 & 0.5 & $7.81 \mathrm{E}+09$ & 12,712 \\
\hline 1 & sample & E1393 & 47 & B2957 & 0.5 & $1.43 E+10$ & 8,168 \\
\hline 1 & sample & E1394 & 75 & B2958 & 0.45 & $1.46 \mathrm{E}+10$ & 7,059 \\
\hline 1 & sample & E1394 & 75 & B2959 & 0.45 & $1.29 E+10$ & 8,447 \\
\hline 1 & blank & E1352 & & B2960 & 0.5 & $2.84 \mathrm{E}+07$ & 342 \\
\hline 1 & sample & E1348 & 26 & B2961 & 0.5 & $5.41 \mathrm{E}+09$ & 36,547 \\
\hline 1 & sample & E1349 & 25 & B2962 & 0.5 & $9.98 \mathrm{E}+09$ & 7,466 \\
\hline 1 & & blank & & B2963 & & $1.59 \mathrm{E}+07$ & 156 \\
\hline 2 & sample & E1467 & 44 & B2980 & 0.5 & $1.03 E+10$ & 35,264 \\
\hline 2 & sample & E1468 & 58 & B2981 & 0.5 & $2.02 \mathrm{E}+10$ & 40,006 \\
\hline 2 & sample & E1469 & 45 & B2982 & 0.5 & $1.53 \mathrm{E}+10$ & 25,167 \\
\hline 2 & blank & E1470 & & B2983 & 0.5 & $3.34 \mathrm{E}+07$ & 362 \\
\hline 2 & sample & E1467 & 44 & B2984 & 0.5 & $1.15 \mathrm{E}+10$ & 33,655 \\
\hline 2 & sample & E1468 & 58 & B2985 & 0.5 & $1.43 \mathrm{E}+10$ & 35,718 \\
\hline 2 & sample & E1469 & 45 & B2986 & 0.5 & $1.74 \mathrm{E}+10$ & 25,957 \\
\hline 2 & sample & E1344 & 31 & B2987 & 0.6 & $2.02 E+10$ & 4,984 \\
\hline 2 & sample & E1345 & 30 & B2988 & 0.5 & $1.23 \mathrm{E}+10$ & 6,725 \\
\hline 2 & sample & E1348 & 26 & B2989 & 0.5 & $9.85 \mathrm{E}+09$ & 44,760 \\
\hline 2 & sample & E1349 & 25 & B2990 & 0.5 & $9.10 E+09$ & 6,323 \\
\hline 2 & sample & E1350 & 34 & B2991 & 0.5 & $8.42 \mathrm{E}+09$ & 4,799 \\
\hline 2 & sample & E1345 & 30 & B2992 & 0.5 & $1.29 \mathrm{E}+10$ & 7,251 \\
\hline 2 & sample & E1350 & 34 & B2993 & 0.5 & 1.11E+10 & 5,606 \\
\hline 2 & & blank & & B2994 & & $9.82 \mathrm{E}+06$ & 168 \\
\hline 3 & pellet E1388 & E1511 & & A1543 & 0.5 & $1.68 \mathrm{E}+08$ & 676 \\
\hline 3 & pellet E1390 & E1512 & & A1544 & 0.5 & $1.09 \mathrm{E}+09$ & 1,333 \\
\hline 3 & pellet E1350 & E1513 & & A1545 & 0.5 & $2.98 \mathrm{E}+08$ & 987 \\
\hline 3 & pellet E1468 & E1514 & & A1546 & 0.5 & $9.06 \mathrm{E}+08$ & 9,757 \\
\hline 3 & pellet E1348 & E1515 & & A154? & 0.5 & $1.49 \mathrm{E}+08$ & 3,313 \\
\hline 3 & sample & E1516 & 50 & A1548 & 0.5 & $1.36 \mathrm{E}+10$ & 28,670 \\
\hline 3 & sample & E1517 & 46 & A1549 & 0.5 & $2.08 \mathrm{E}+10$ & 7,867 \\
\hline 3 & sample & E1518 & 50 & A1550 & 0.5 & $2.27 E+10$ & 7,076 \\
\hline 3 & sample & E1519 & 48 & A1551 & 0.5 & $1.54 \mathrm{E}+10$ & 4,852 \\
\hline 3 & sample & E1520 & 50 & A1552 & 0.5 & $2.42 \mathrm{E}+10$ & 11,749 \\
\hline 3 & sample & E1521 & 50 & A1553 & 0.5 & $1.67 \mathrm{E}+10$ & 140,535 \\
\hline 3 & sample & E1522 & 62 & A1554 & 0.5 & 1.19E+10 & 47,557 \\
\hline 3 & sample & E1523 & 46 & A1555 & 0.5 & $1.56 \mathrm{E}+10$ & 9,972 \\
\hline 3 & blank & E1524 & & A1556 & 0.5 & $3.87 \mathrm{E}+07$ & 719 \\
\hline 3 & & blank & & A1557 & & $1.85 \mathrm{E}+07$ & not sequenced \\
\hline 4 & & blank & & A2001 & & $3.32 \mathrm{E}+07$ & 153 \\
\hline 4 & pellet E1388 & E1511 & & A2002 & 0.5 & $1.32 E+08$ & 487 \\
\hline 4 & pellet E1390 & E1512 & & A2003 & 0.5 & $5.35 \mathrm{E}+08$ & 697 \\
\hline 4 & pellet E1350 & E1513 & & A2004 & 0.5 & $1.48 \mathrm{E}+08$ & 448 \\
\hline 4 & pellet E1468 & E1514 & & A2005 & 0.5 & $3.79 \mathrm{E}+08$ & 3,951 \\
\hline 4 & pellet E1348 & E1515 & & A2006 & 0.5 & $7.61 \mathrm{E}+07$ & 1,196 \\
\hline 4 & sample & E1516 & 50 & A2007 & 0.5 & $3.23 E+09$ & 12,219 \\
\hline 4 & sample & E1517 & 46 & A2008 & 0.5 & $3.81 \mathrm{E}+09$ & 3,193 \\
\hline 4 & sample & E1518 & 50 & A2009 & 0.5 & $5.75 \mathrm{E}+09$ & 2,524 \\
\hline 4 & sample & E1519 & 48 & A2010 & 0.5 & $4.30 \mathrm{E}+09$ & 1,812 \\
\hline 4 & sample & E1520 & 50 & A2011 & 0.5 & $7.29 E+09$ & 4,940 \\
\hline 4 & sample & E1521 & 50 & A2012 & 0.5 & $8.42 \mathrm{E}+09$ & 74,091 \\
\hline 4 & sample & E1522 & 62 & A2013 & 0.5 & $3.72 \mathrm{E}+09$ & 14,340 \\
\hline 4 & sample & E1523 & 46 & A2014 & 0.5 & $4.69 \mathrm{E}+09$ & 3,542 \\
\hline 4 & blank & E1524 & & A2015 & 0.5 & $4.79 \mathrm{E}+07$ & 372 \\
\hline 5 & & blank & & A2016 & & $9.30 \mathrm{E}+06$ & 203 \\
\hline 5 & sample & E1534 & 47 & A2017 & 0.6 & $1.26 \mathrm{E}+10$ & 4,335 \\
\hline 5 & sample & E1535 & 47 & A2018 & 0.6 & $6.81 \mathrm{E}+09$ & 3,437 \\
\hline 5 & sample & E1536 & 50 & A2019 & 0.6 & $4.09 \mathrm{E}+09$ & 4,464 \\
\hline 5 & sample & E1537 & 53 & A2020 & 0.6 & 1.18E+10 & 6,371 \\
\hline 5 & sample & E1538 & 63 & A2021 & 0.6 & $5.73 E+09$ & 2,921 \\
\hline 5 & sample & E1539 & 50 & A2022 & 0.6 & $7.89 \mathrm{E}+09$ & 4,648 \\
\hline 5 & sample & E1540 & 43 & A2023 & 0.6 & $9.56 \mathrm{E}+09$ & 5,679 \\
\hline 5 & sample & E1541 & 49 & A2024 & 0.6 & $4.14 \mathrm{E}+09$ & 18,565 \\
\hline 5 & sample & E1542 & 44 & A2025 & 0.6 & $4.81 \mathrm{E}+09$ & 18,168 \\
\hline 5 & sample & E1543 & 43 & A2026 & 0.6 & $1.00 \mathrm{E}+10$ & 15,328 \\
\hline 5 & surface & E1544 & 56 & A2027 & 0.6 & $4.37 E+09$ & 132,351 \\
\hline 5 & surface & E1545 & 65 & A2028 & 0.6 & $1.09 \mathrm{E}+10$ & 734,511 \\
\hline 5 & pellet E1522 & E1546 & & A2029 & 0.6 & $6.70 \mathrm{E}+08$ & 8,024 \\
\hline 5 & blank & E1547 & & A2030 & 0.6 & $2.39 \mathrm{E}+07$ & 309 \\
\hline 6 & & blank & & A2031 & & $4.92 \mathrm{E}+06$ & 122 \\
\hline 6 & sample & E1534 & 47 & A2032 & 0.4 & $6.06 \mathrm{E}+09$ & 3,810 \\
\hline 6 & sample & E1535 & 47 & A2033 & 0.4 & $3.08 \mathrm{E}+09$ & 2,320 \\
\hline 6 & sample & E1536 & 50 & A2034 & 0.4 & $3.08 E+09$ & 3,461 \\
\hline 6 & sample & E1537 & 53 & A2035 & 0.4 & $5.04 \mathrm{E}+09$ & 3,553 \\
\hline 6 & sample & E1538 & 63 & A2036 & 0.4 & $3.20 \mathrm{E}+09$ & 1,529 \\
\hline 6 & sample & E1539 & 50 & A2037 & 0.4 & $1.92 \mathrm{E}+09$ & 1,629 \\
\hline 6 & sample & E1540 & 43 & A2038 & 0.4 & $2.88 \mathrm{E}+09$ & 2,157 \\
\hline 6 & sample & E1541 & 49 & A2039 & 0.4 & $7.59 \mathrm{E}+09$ & 20,365 \\
\hline 6 & sample & E1542 & 44 & A2040 & 0.4 & $3.25 \mathrm{E}+09$ & 11,083 \\
\hline 6 & sample & E1543 & 43 & A2041 & 0.4 & $6.07 \mathrm{E}+09$ & 9,430 \\
\hline 6 & surface & E1544 & 56 & A2042 & 0.4 & $8.83 E+09$ & 226,317 \\
\hline 6 & surface & E1545 & 65 & A2043 & 0.4 & $1.71 \mathrm{E}+10$ & 773,319 \\
\hline 6 & pellet E1522 & E1546 & & A2044 & 0.4 & $5.01 \mathrm{E}+08$ & 6,435 \\
\hline 6 & blank & E1547 & & A2045 & 0.4 & $1.02 \mathrm{E}+07$ & 262 \\
\hline
\end{tabular}

Substitution frequencies are not reported (N.R.) if based on fewer than 100 observations. 
Extended Data Table 2 | Results from shallow shotgun sequencing of a subset of libraries

\begin{tabular}{|c|c|c|c|c|c|c|}
\hline $\begin{array}{c}\text { Extracted } \\
\text { material }\end{array}$ & $\begin{array}{l}\text { library } \\
\text { ID }\end{array}$ & $\begin{array}{l}\text { total number } \\
\text { of sequences } \\
\text { (overlap- } \\
\text { merged, } \\
>=35 \mathrm{bp} \text { ) } \\
\end{array}$ & $\begin{array}{c}\text { mapped } \\
\text { sequences } \\
\text { (map quality } \\
>=30 \text { ) }\end{array}$ & $\begin{array}{c}\text { fraction } \\
\text { mapped } \\
{[\%]}\end{array}$ & $\begin{array}{c}\text { 5' C to } \mathrm{T} \\
\text { substitution } \\
\text { frequency [\%] } \\
\text { (observations) }\end{array}$ & $\begin{array}{c}\text { 3' } \mathrm{C} \text { to } \mathrm{T} \\
\text { substitution } \\
\text { frequency [\%] } \\
\text { (observations) }\end{array}$ \\
\hline pellet E1388 & A1543 & 330,819 & 535 & 0.162 & $1(1 / 110)$ & $4(3 / 70)$ \\
\hline pellet E1390 & A1544 & 609,472 & 200 & 0.033 & $0(0 / 45)$ & $0(0 / 27)$ \\
\hline pellet E1350 & A1545 & 382,645 & 307 & 0.080 & $0(0 / 63)$ & $6(3 / 51)$ \\
\hline pellet E1468 & A1546 & 562,458 & 1,375 & 0.244 & $3(7 / 248)$ & $2(4 / 180)$ \\
\hline pellet E1348 & A1547 & 382,039 & 794 & 0.208 & $1(2 / 141)$ & $1(1 / 126)$ \\
\hline sample & A1548 & 569,592 & 535 & 0.094 & $1(1 / 104)$ & $2(1 / 54)$ \\
\hline sample & A1549 & 383,570 & 82 & 0.021 & $5(1 / 19)$ & $10(1 / 10)$ \\
\hline sample & A1550 & 556,828 & 45 & 0.008 & $0(0 / 6)$ & $0(0 / 2)$ \\
\hline sample & A1551 & 521,648 & 60 & 0.012 & $0(0 / 10)$ & $0(0 / 6)$ \\
\hline sample & A1552 & 492,761 & 107 & 0.022 & $10(2 / 20)$ & $0(0 / 9)$ \\
\hline sample & A1553 & 391,037 & 2,575 & 0.659 & $1(7 / 477)$ & $3(8 / 301)$ \\
\hline sample & A1554 & 522,604 & 934 & 0.179 & $3(5 / 185)$ & $6(6 / 102)$ \\
\hline sample & A1555 & 439,135 & 73 & 0.017 & $0(0 / 14)$ & $29(2 / 7)$ \\
\hline pellet E1388 & A2002 & 80,590 & 111 & 0.138 & $0(0 / 22)$ & $0(0 / 9)$ \\
\hline pellet E1390 & A2003 & 176,798 & 47 & 0.027 & $0(0 / 6)$ & $0(0 / 5)$ \\
\hline pellet E1350 & A2004 & 80,519 & 86 & 0.107 & $0(0 / 12)$ & $8(1 / 12)$ \\
\hline pellet E1468 & A2005 & 153,555 & 441 & 0.287 & $0(0 / 78)$ & $0(0 / 52)$ \\
\hline pellet E1348 & A2006 & 21,876 & 41 & 0.187 & $0(0 / 6)$ & $0(0 / 3)$ \\
\hline sample & A2007 & 198,123 & 169 & 0.085 & $0(0 / 38)$ & $0(0 / 26)$ \\
\hline sample & A2008 & 182,797 & 44 & 0.024 & $0(0 / 8)$ & $0(0 / 5)$ \\
\hline sample & A2009 & 271,679 & 19 & 0.007 & $0(0 / 3)$ & $0(0 / 3)$ \\
\hline sample & A2010 & 222,503 & 23 & 0.010 & $0(0 / 6)$ & $0(0 / 2)$ \\
\hline sample & A2011 & 193,697 & 50 & 0.026 & $0(0 / 8)$ & $0(0 / 5)$ \\
\hline sample & A2012 & 239,484 & 1,559 & 0.651 & $1(4 / 291)$ & $3(5 / 187)$ \\
\hline sample & A2013 & 171,305 & 308 & 0.180 & $2(1 / 51)$ & $0(0 / 26)$ \\
\hline sample & A2014 & 242,819 & 43 & 0.018 & $11(1 / 9)$ & $0(0 / 6)$ \\
\hline sample & A2017 & 172,196 & 21 & 0.012 & $0(0 / 5)$ & $0(0 / 4)$ \\
\hline sample & A2018 & 182,640 & 33 & 0.018 & $0(0 / 6)$ & $22(2 / 9)$ \\
\hline sample & A2019 & 241,673 & 50 & 0.021 & $0(0 / 7)$ & $17(1 / 6)$ \\
\hline sample & A2020 & 188,983 & 51 & 0.027 & $10(1 / 10)$ & $0(0 / 6)$ \\
\hline sample & A2021 & 148,940 & 21 & 0.014 & $50(1 / 2)$ & $50(1 / 2)$ \\
\hline sample & A2022 & 179,754 & 52 & 0.029 & $0(0 / 13)$ & $0(0 / 4)$ \\
\hline sample & A2023 & 169,802 & 64 & 0.038 & $0(0 / 8)$ & $0(0 / 9)$ \\
\hline sample & A2024 & 171,019 & 240 & 0.140 & $0(0 / 51)$ & $0(0 / 25)$ \\
\hline sample & A.2025 & 200,408 & 462 & 0.231 & $1(1 / 75)$ & $2(1 / 61)$ \\
\hline sample & A2026 & 158,056 & 130 & 0.082 & $0(0 / 25)$ & $0(0 / 16)$ \\
\hline surface & A2027 & 204,421 & 3,977 & 1.945 & $2(14 / 814)$ & $3(12 / 353)$ \\
\hline surface & A2028 & 210,913 & 17,647 & 8.367 & $2(55 / 3379)$ & $3(49 / 1790)$ \\
\hline pellet E1522 & A2029 & 178,297 & 654 & 0.367 & $0(0 / 129)$ & $0(0 / 74)$ \\
\hline sample & A2032 & 172,846 & 24 & 0.014 & $0(0 / 2)$ & $0(0 / 2)$ \\
\hline sample & A2033 & 159,571 & 11 & 0.007 & $0(0 / 1)$ & $0(0 / 0)$ \\
\hline sample & A2034 & 194,795 & 36 & 0.018 & $25(2 / 8)$ & $0(0 / 4)$ \\
\hline sample & A2035 & 196,685 & 62 & 0.032 & $8(1 / 12)$ & $0(0 / 10)$ \\
\hline sample & A2036 & 121,160 & 23 & 0.019 & $0(0 / 7)$ & $20(1 / 5)$ \\
\hline sample & A2037 & 158,477 & 45 & 0.028 & $0(0 / 8)$ & $0(0 / 2)$ \\
\hline sample & A2038 & 212,279 & 75 & 0.035 & $0(0 / 20)$ & $14(1 / 7)$ \\
\hline sample & A2039 & 185,602 & 247 & 0.133 & $0(0 / 47)$ & $3(1 / 32)$ \\
\hline sample & A2040 & 147,584 & 365 & 0.247 & $2(1 / 61)$ & $0(0 / 47)$ \\
\hline sample & A2041 & 174,738 & 168 & 0.096 & $3(1 / 30)$ & $15(3 / 20)$ \\
\hline surface & A2042 & 194,501 & 3,522 & 1.811 & $1(6 / 666)$ & $2(9 / 422)$ \\
\hline surface & A2043 & 189,332 & 14,305 & 7.556 & $1(34 / 2770)$ & $2(44 / 1903)$ \\
\hline pellet E1522 & A2044 & 167,925 & 534 & 0.318 & $2(2 / 101)$ & $0(0 / 71)$ \\
\hline
\end{tabular}


Extended Data Table 3 | Number of sequences retained in the sample libraries after each step of processing and filtering

\begin{tabular}{lrrr}
\hline \multicolumn{1}{c}{ Filter } & $\begin{array}{c}\text { Enrichment with } \\
\text { human capture } \\
\text { probes }\end{array}$ & $\begin{array}{c}\text { Enrichment with } \\
\text { Denisovan capture } \\
\text { probes }\end{array}$ & Combined \\
\hline All sequences & $402,938,161$ & $154,005,123$ & $556,943,284$ \\
Overlap-merged & $367,593,202$ & $146,852,057$ & $514,445,259$ \\
Length >= 30bp & $320,441,187$ & $124,694,112$ & $445,135,299$ \\
Mapped & $21,634,872$ & $34,388,775$ & $56,023,647$ \\
Unique & $2,171,801$ & & $2,805,919$ \\
In libraries with terminal C to T substitution & 219,766 & & 299,093 \\
$\quad$ frequencies >= 5.0\% & 197,019 & & 264,319 \\
$<=2$ mismatches other than C to T & 196,536 & & 263,302 \\
Mapping quality >=30 & 82,239 & & 105,070 \\
Length <= 45 & & & $10,160 / 15,528$ \\
Terminal C to T substitution / C to T substitution & 6,706 & & \\
$\quad$ in first or last three alignment positions & & & \\
\hline
\end{tabular}

Sequences obtained from the blank controls are not included. All filters are additive in the order indicated. 
Extended Data Table 4 Inferred time to the most recent common ancestor (TMRCA) of the modern human, Neanderthal, chimpanzee and bonobo mtDNAs, as well as divergence estimates for human/chimpanzee and bonobo/chimpanzee mtDNA (continuation of Table 1)

Divergence dates and molecular age estimates in million years before present [95\% HPD interval in brackets]

\begin{tabular}{lccc} 
Mitochondrial lineages & \multicolumn{3}{c}{$[95 \%$ HPD interval in brackets $]$} \\
\cline { 2 - 4 } & Strict filters & Inclusive filters & $\begin{array}{c}\text { Strict filters, enriched } \\
\text { with human probes only }\end{array}$ \\
\hline TMRCA modern humans & $0.21[0.15-0.29]$ & $0.21[0.15-0.30]$ & $0.18[0.12-0.25]$ \\
TMRCA Neanderthals & $0.15[0.11-0.20]$ & $0.15[0.11-0.19]$ & $0.13[0.10-0.17]$ \\
TMRCA chimpanzees & $0.86[0.59-1.16]$ & $0.89[0.61-1.22]$ & $0.73[0.48-1.04]$ \\
TMRCA bonobos & $0.37[0.24-0.50]$ & $0.36[0.24-0.50]$ & $0.30[0.19-0.43]$ \\
Human - Chimpanzee & $6.18[4.33-8.45]$ & $6.44[4.40-8.82]$ & $5.43[3.54-7.64]$ \\
Chimpanzee - Bonobo & $1.83[1.27-2.48]$ & $1.91[1.30-2.61]$ & $1.61[1.06-2.27]$ \\
\hline
\end{tabular}

